\title{
BMJ Open Association of co-prescribing of opioid and benzodiazepine substitutes with incident falls and fractures among older adults: a cohort study
}

\author{
Rahul Shah (D) , ${ }^{1}$ Mukaila A Raji (D) , ${ }^{2,3}$ Jordan Westra, ${ }^{4}$ Yong-Fang Kuo ${ }^{3,5}$
}

To cite: Shah R, Raji MA, Westra J, et al. Association of co-prescribing of opioid and benzodiazepine substitutes with incident falls and fractures among older adults: a cohort study. BMJ Open 2021;11:e052057. doi:10.1136/ bmjopen-2021-052057

- Prepublication history and additional supplemental material for this paper are available online. To view these files, please visit the journal online (http://dx.doi.org/10.1136/ bmjopen-2021-052057).

Received 06 April 2021 Accepted 13 December 2021

Check for updates

(C) Author(s) (or their employer(s)) 2021. Re-use permitted under CC BY-NC. No commercial re-use. See rights and permissions. Published by BMJ.

For numbered affiliations see end of article.

Correspondence to

Dr Rahul Shah;

Rahul.shah@vumc.org

\section{ABSTRACT}

Objective Examine the association between the coprescribing of opioids, benzodiazepines, gabapentinoids (pregabalin and gabapentin) and selective serotonin reuptake inhibitors/serotonin and norepinephrine reuptake inhibitors (SSRI/SNRIs) in different combinations and the risk of falls and fractures.

Design Retrospective cohort study from 2015 to 2018.

Setting Medicare enrolment and claims data.

Participants Medicare beneficiaries with both chronic pain and anxiety disorders in 2016 with continuous enrolments in Parts A and B from 2015 to 2016 who were prescribed any combination of opioid, benzodiazepine, gabapentinoid and SSRI/SNRI in 2017 for $\geq 7$ days, as documented in their Medicare Part D coverage.

Interventions Any combination of use of seven drug regimens (benzodiazepine +opioid; benzodiazepine + gabapentinoid; benzodiazepine +SSRI/ SNRI; opioid +gabapentinoid; opioid +SSRI/SNRI; gabapentinoid +SSRI/SNRI; $\geq 3$ drug classes).

Main outcomes First event of fall and the first event of fracture after the index date, which was the first day of combination drug use that lasted $\geq 7$ days in 2017.

Results A total of 47964 patients (mean [SD] age, 75.9 [7.1]; 78.0\% woman) with diagnoses of both chronic pain and anxiety were studied. The median (Q1-Q3) duration of drug combination use was 26 (14-30) days. After adjusting for demographic characteristics, chronic conditions and history of hospitalisation and fall or fracture, the coprescribing of $\geq 3$ drugs (adjusted HR [aHR], $1.38 ; 95 \% \mathrm{Cl}$ 1.14 to 1.67 ) and opioid plus gabapentinoid (aHR, 1.18; $95 \% \mathrm{Cl} 1.02$ to 1.37 ) were associated with a high fall risk, compared with benzodiazepineplus opioid co-prescribing, findings consistent with the secondary analysis using inverse probability of treatment weighting with propensity scores. The co-prescribing of benzodiazepine plus gabapentinoid (aHR, $0.76 ; 95 \% \mathrm{Cl} 0.59$ to 0.98 ) was associated with lower fracture risk compared with the co-prescribing of benzodiazepine plus opioid, though this finding was not robust.

Conclusions Our findings add to comparative toxicity research on different combinations of gabapentinoids and serotonergic agents commonly prescribed with or as substitutes for opioids and benzodiazepines in patients with co-occurring chronic pain and anxiety.
Strengths and limitations of this study

- This study is a retrospective cohort study with a large sample size of 47964 Medicare beneficiaries.

- This study evaluates fall and fracture outcomes in patients with both chronic pain and anxiety disorders, two conditions that commonly co-exist in realworld clinical practice.

- Fall and fracture outcomes were compared across subsets of patients who were prescribed seven different drug combinations commonly used to treat chronic pain and anxiety, adding to the limited comparative toxicity research of combinations of opioids, benzodiazepines, gabapentinoids and selective serotonin reuptake inhibitors/serotonin norepinephrine reuptake inhibitors.

- Multivariable models accounting for demographic and clinical characteristics were used to evaluate outcomes, and inverse probability of treatment weighting with propensity score analyses was used to test for robustness of findings.

- One limitation is that the subsets of patients prescribed different drug combinations may differ in regards to their inherent fall risk, thus altering clinicians' decision to prescribe certain medications, that is, confounding by indication.

\section{INTRODUCTION}

The rise in opioid prescribing in the USA over the last two decades has been associated with concomitant increases in the rates of opioid use disorder, overdose and mortality. ${ }^{1-3}$ Approximately one-third of opioid overdose deaths involve co-use of benzodiazepines (benzos), ${ }^{45}$ a class of medication commonly prescribed for anxiety and sleep disorders. ${ }^{4-6}$ The co-prescribing of opioids and benzos has steadily risen in recent years, ${ }^{5-9}$ a reflection in part of the high rate of co-occurrence of pain and anxiety disorders. ${ }^{10}$ In a retrospective analysis of claims data from 315428 commercially insured patients aged 18-64, Sun et a $\hat{\theta}^{9}$ reported that $9 \%$ used both an opioid and benzo in 2001, increasing to $17 \%$ in 2013. 
Concurrent opioid and benzodiazepine use increases opioid overdose risk because of their synergistic depression of the central nervous system (CNS) and respiratory function. ${ }^{11}{ }^{12}$ Concurrent opioid and benzodiazepine use is also associated with increased risk of emergency room visit,${ }^{9}$ inpatient admission, ${ }^{9}$ falls, ${ }^{13}{ }^{14}$ fractures ${ }^{13} 14$ and death due to opioid overdose. ${ }^{5}$

In response, the 2016 Centers for Disease Control and Prevention (CDC) opioid prescribing guideline recommended that clinicians avoid co-prescribing opioids and benzodiazepines 'whenever possible'. ${ }^{15}$ The Federal Drug Administration (FDA) has also strongly cautioned against their co-prescribing. ${ }^{16}$ These strategies and other government, health system and payer policies have been associated with a decrease in the rate of opioid and benzodiazepine prescribing, separately and concurrently. ${ }^{17} 18$ Restrictions on opioid prescribing have paralleled greater interest in the prescribing of the antiepileptic drugs gabapentinoids (GABA), ie, gabapentin and pregabalin as non-opioid analgesics. ${ }^{19-21}$ Data from a nationally representative sample of 346177 Americans showed that GABA prescribing has increased from $1.2 \%$ in 2002 to $3.9 \%$ in $2015 .{ }^{21}$ While FDA approved for a limited number of pain conditions, GABAs are widely used off-label to treat chronic pain despite limited evidence of efficacy and safety. ${ }^{19} 2122$ Other off-label use includes prescribing for anxiety disorders. ${ }^{23}{ }^{24}$ The premise for a shift to GABA as a safer alternative to opioids is not supported by evidence, as recent data showed associations of GABA use with dizziness, sedation, falls, fractures and overdose. 1920222526

While data have shown a shift towards GABA prescribing as a non-opioid analgesic after the 2016 CDC guideline, a similar change in the rate of non-benzodiazepine alternatives (selective serotonin reuptake inhibitors [SSRIs] and serotonin and norepinephrine reuptake inhibitors [SNRIs]) for treating comorbid anxiety in chronic pain patients is not clear. Modern evidence-informed prescribing guidelines strongly recommend SSRIs and SNRIs as first-line anxiolytic agents over benzodiazepines because of their better safety and efficacy profile than benzos. ${ }^{27-29}$ SNRIs have an additional FDA indication for neuropathic pain and are commonly used for this purpose. ${ }^{30} 31$ Unknown, however, are the differences in toxicity outcomes (eg, falls and fractures, overdose, hospitalisation, death) between opioid/benzo coprescribing and opioid-sparing or benzodiazepine-sparing combinations, such as those that use gabapentinoids or SSRI/ SNRIs.

Understanding these differences is especially important for older adults at higher risk of adverse events from the co-use of multiple psychoactive medications. Minimising falls and fractures is a key consideration when prescribing multiple medications for older adults. Comparative safety data can help clinicians choose combinations with the least toxicity to safely treat patients with chronic pain and anxiety disorders, two conditions that commonly co-occur in real-world clinical practice. ${ }^{10}$ To address these knowledge gaps, this study aims to examine the association between seven drug combinations commonly used to treat chronic pain and anxiety disorders and the risk of falls and fractures.

\section{METHODS}

\section{Data source}

This was a retrospective cohort study using enrolment and claims data from a 20\% national sample of Medicare beneficiaries enrolled from 2015 to 2018. The data source included the Master Beneficiary Summary File (MBSF), Medicare Provider Analysis and Review (MEDPAR) file, Outpatient Standard Analytic File (OUTSAF), and Carrier and Prescription Drug Event files. The institutional review board of the University of Texas Medical Branch approved this study.

\section{Study cohort}

The cohort selection flowchart is shown in online supplemental table 1 . We selected Medicare beneficiaries aged 65 and older who were diagnosed with both chronic pain and anxiety in 2016; continuously enrolled in Parts A and B from 2015 to 2016; used some combination of opioid, benzo, SSRI/SNRI or GABA classes of medication in 2017 for at least 7 days; had 1 month Part $\mathrm{D}$ coverage prior to beginning the combination use; no prior combination use in the prior 30 days to initiation of a combination and had complete information in the data files. Chronic pain and anxiety were ascertained using the Chronic Conditions Data Warehouse (CCW) condition categories (online supplemental table 2). These categories are created by including beneficiaries with at least one inpatient diagnosis or two non-drug claims of any type in the 2-year period. ${ }^{32}$

\section{Measurements}

Combination drug use

Combination of drug use was created by assessing which of the outpatient prescription drugs each beneficiary was using on each day of 2017. The first combination that was used 7 or more days in 2017 was used as the index combination; the first day of this combination use was the index date. If participants used a combination for $\geq 7$ days in 2017, they were followed into 2018 for complete prescription duration and outcome data. The index combination was categorised into seven groups: benzo plus opioid (benzo +opioid); benzo plus GABA (benzo +GABA); benzo plus SSRI or SNRI (benzo +SSRI/ SNRI); opioid plus GABA (opioid +GABA); opioid plus SSRI or SNRI (opioid +SSRI/SNRI); GABA plus SSRI or SNRI (GABA +SSRI/SNRI) and $\geq 3$ drugs from the four medication classes. We grouped SSRI/SNRI together as they share mechanisms of action. To ensure that the index combination was an initiation of combination, patients with any of the seven study combinations in the 30 days before the index date were excluded. The list of medications for each drug class is included in online supplemental table 3 . 
Fall and fracture

The study outcomes were (1) the first event of falls as any diagnosis and (2) the first event of fracture as a primary diagnosis after the index date. Falls and fractures were assessed using diagnosis codes from the Tenth Revision of the International Statistical Classification of Diseases and Related Health Problems (online supplemental table 4). Binary indicators were created for each of the outcomes and time to the first event was assessed from the index date. The median and interquartile duration of the initial drug combination was 26 (14-30) days (table 1). Therefore, patients were censored 30 days after the end of their initial drug combination, at loss of Medicare coverage, death or at the end of 6-month follow-up from the drug combination initiation.

\section{Covariates}

Sex, age category, race/ethnicity, Medicaid enrolment, US Census region, original reason for Medicare entitlement, history of fall or fracture, history of hospitalisation and chronic conditions were adjusted for in the analysis. Demographic variables were taken from the MBSF; history of fall, fracture or hospitalisation in the 12 months before the index date were created from MEDPAR, OUTSAF and Carrier files; and chronic conditions were taken from the CCW categories. We selected 19 chronic conditions related to the study outcomes: alcohol use disorders, Alzheimer's disease/dementia, arthritis, asthma, cancer (breast, colorectal, endometrial, lung, leukaemia, lymphoma), chronic kidney diseases (CKD), chronic obstructive pulmonary disease (COPD), depression, diabetes, drug use disorder, epilepsy, hearing impairment, liver disease, migraine/headache, mobility impairment, obesity, osteoporosis, spine injury and vision impairment.

\section{Statistical analysis}

Descriptive statistics were generated for each of the covariates with mean and SD for numeric variables and count and per cent for categorical variables across seven drug combination groups. The unadjusted fall or fracture rates for each group were estimated using the Kaplan-Meier method. Cox proportional hazard models were used to assess the effect of drug combination use on each of the outcomes separately adjusted for all variables. In addition, prespecified interactions were assessed in the model adjusted for all variables between drug combination and age, Alzheimer's/dementia status for both outcomes and history of fall/fracture for their correspondent outcome. Proportionality of hazards was tested by adding to the model the logarithm of the time to fall or fracture and assessing its significance. Furthermore, propensity score was generated with a multinomial logit model using average treatment effect estimation that considered all covariates listed in table 1. Backward elimination was used to select variables included in the propensity model, eventually eliminating the comorbidities of asthma and liver disease. Then we used inverse probability of treatment weighting (IPTW) with propensity scores in Cox proportional hazard models to examine the effect of drug combination on outcomes and limit the effect of confounding by limitation across the study groups. Absolute standardised differences were used to assess balance across treatment groups. Because all variables were balanced following IPTW propensity score, no additional variables were included in the proportional hazards models. Finally, we conducted two sets of sensitivity analyses. First, we excluded patients with cancer diagnoses from our main analyses. Second, to better identify new initiation of drug combination, we repeated our main analyses with a 3-month lookback period, which included patients with part $\mathrm{D}$ coverage in the 3 months prior to beginning the combination use and did not have any studied combination in these 3 months. The sensitivity analyses were adjusted for all demographic and clinical characteristics. All analyses were performed with SAS Enterprise V.7.12 at the Centers for Medicare and Medicaid Services Virtual Research Data Center (SAS Institute, Cary, North Carolina).

\section{Patient and public involvement}

Patients and the public were not involved in the design, conduct, reporting or dissemination plans of our research. The results of our study are not planned to be disseminated directly to study participants, as our data source is deidentified clinical data. However, this report will be available open access to all patient and clinician stakeholders.

\section{RESULTS}

\section{Baseline characteristics}

A total of 47964 Medicare beneficiaries with diagnoses of both chronic pain and anxiety who used any of the seven study drug combinations were included in this study. Table 1 presents the baseline characteristics of these individuals, stratified by the drug combinations. Of the full cohort, $10261(21.4 \%)$ used the combination of benzo +opioid, $9541(19.9 \%)$ used benzo +GABA, $4420(9.2 \%)$ used benzo +SSRI/SNRI, 8625 (18.0\%) used opioid +GABA, $6843(14.3 \%)$ used opioid +SSRI/ SNRI, 5499 (11.5\%) used GABA +SSRI/SNRI and 2775 $(5.8 \%)$ used any combination of $\geq 3$ drugs from the four drug classes. Of those who used $\geq 3$ drug classes, the most common combination was benzo +opioid+SSRI/SNRI $(36 \%)$, followed by opioid +SSRI/SNRI+GABA $(22 \%)$, benzo +opioid+ GABA (19\%), benzo +SSRI/SNRI+GABA $(16 \%)$ and benzo +opioid+SSRI/SNRI+GABA $(7 \%)$. The median (Q1-Q3) duration of drug combination use was 26 (14-30) days and mean (SD) was 32.0 (32.3) days. Overall, most individuals were women $(78.0 \%)$ and white $(87.6 \%)$, and the mean age was $75.9(\mathrm{SD}, 7.1)$ years. These characteristics were similar across the seven drug combination groups. A high proportion of the total study cohort had the comorbid chronic conditions of arthritis $(78.2 \%)$, depression $(62.3 \%)$, diabetes $(37.4 \%)$, CKD 


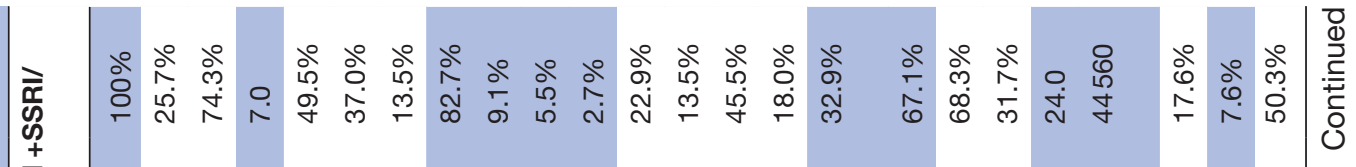

总䍃

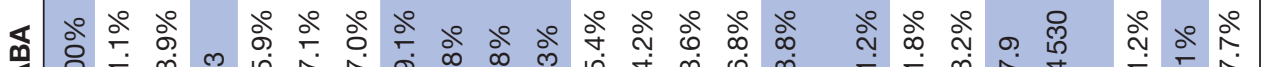

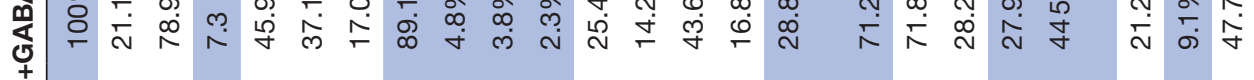

:

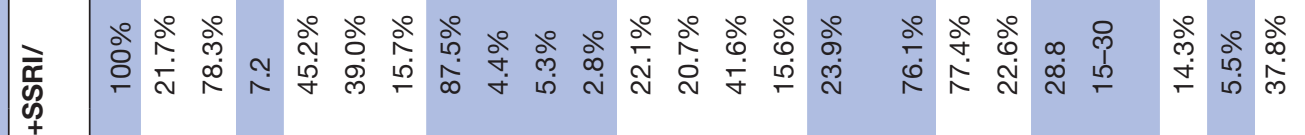

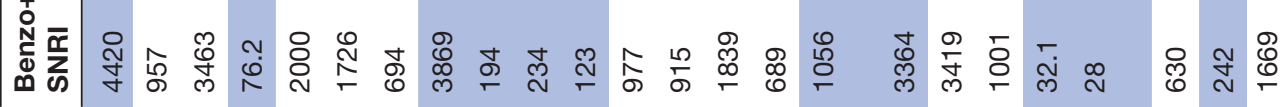

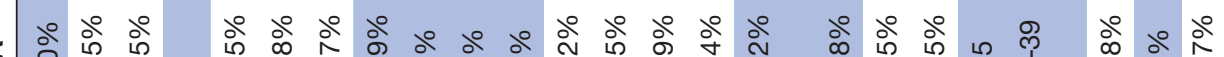

荇

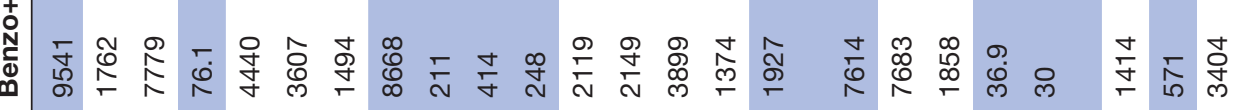

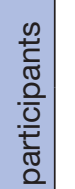

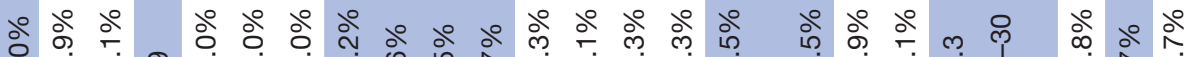

$\circ$

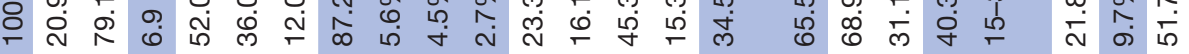

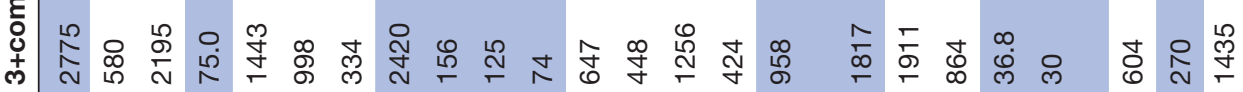

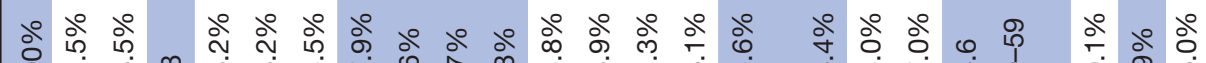
㭊

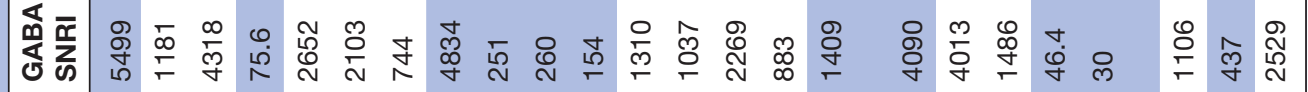

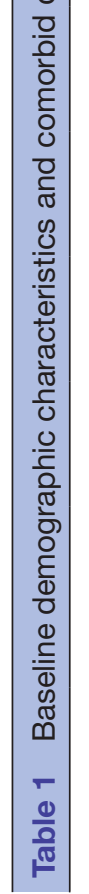

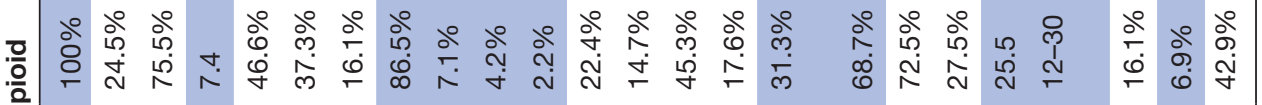

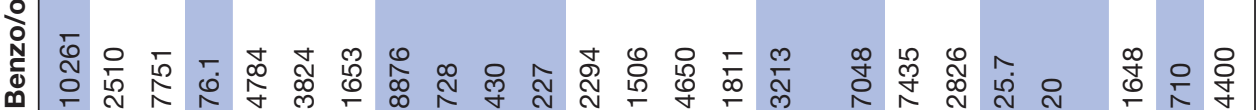

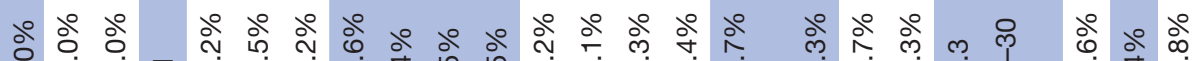

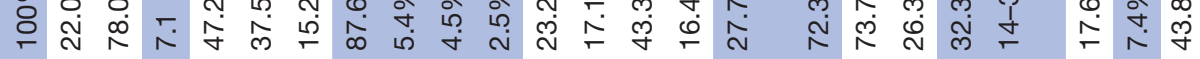

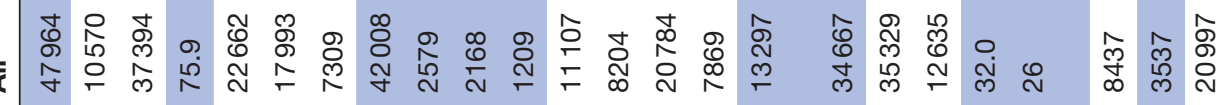

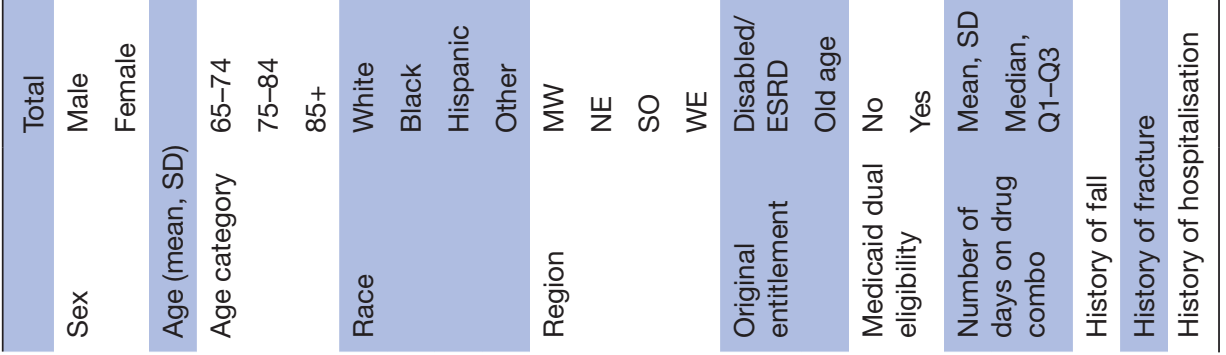




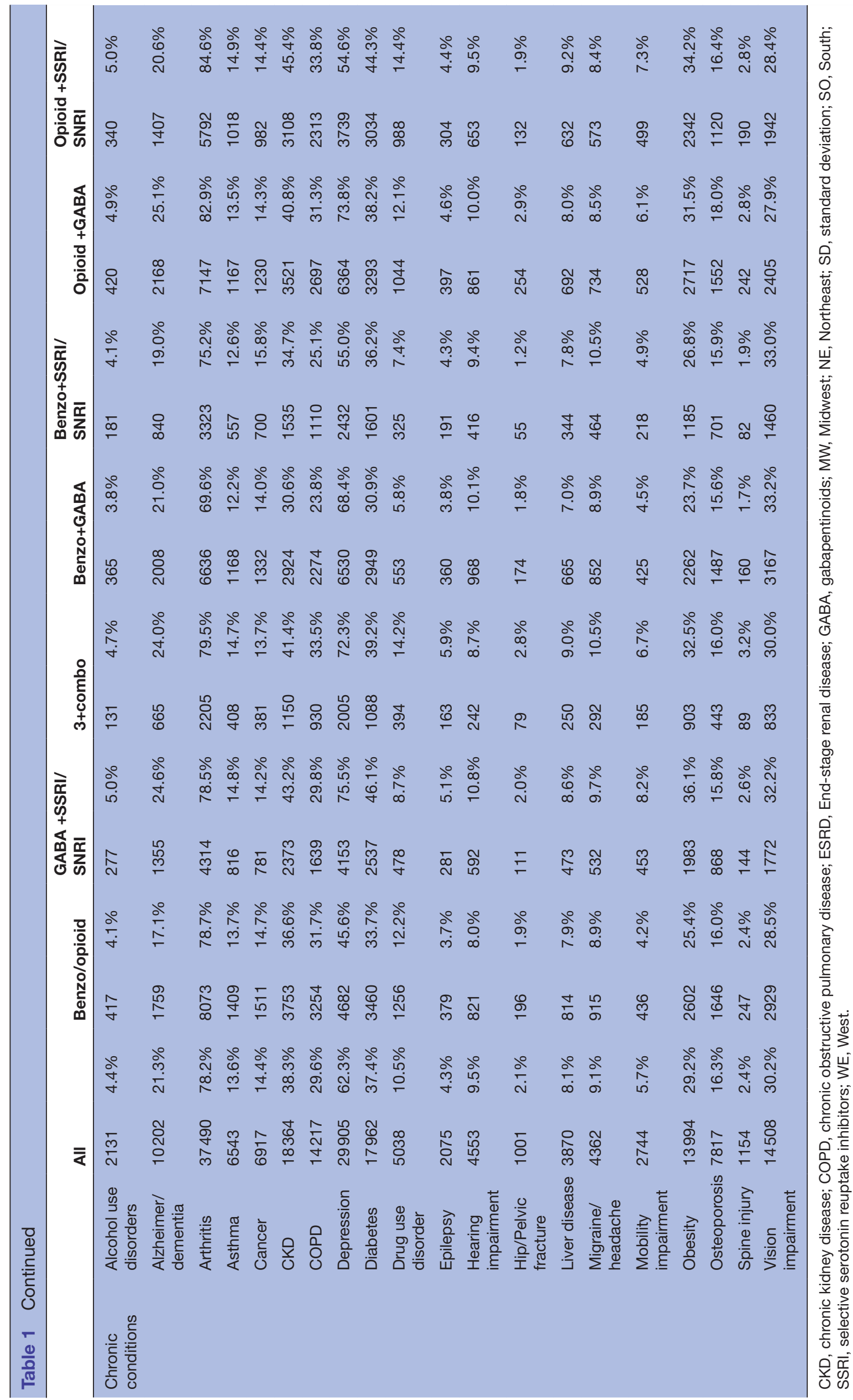


$(38.3 \%)$, vision impairment $(30.2 \%)$ and obesity $(29.2 \%)$. Some groups that received different drug combinations differed in demographic characteristics and comorbid conditions. For example, some patient-level differences for those prescribed benzo +GABA versus opioid +SSRI/ SNRI were gender $(81.5 \%$ vs $74.3 \%$ female, respectively), Medicaid dual eligibility (19.5\% vs $31.7 \%$ ) and comorbid arthritis $(69.6 \%$ vs $84.6 \%)$.

\section{Fall and fracture event analysis}

The estimated cumulative risks for falls for the seven drug combinations over a 6-month period are presented as incidence curves in figure 1A (benzo +opioid: $10.1 \%$ at 6 months; benzo +GABA: $9.8 \%$; benzo +SSRI/SNRI: 9.2\%; opioid +GABA: $11.7 \%$; opioid +SSRI/SNRI: $12.2 \%$; GABA +SSRI/SNRI: $10.2 \%$; $\geq 3$ drugs: $13.7 \%$ ). Figure 1B
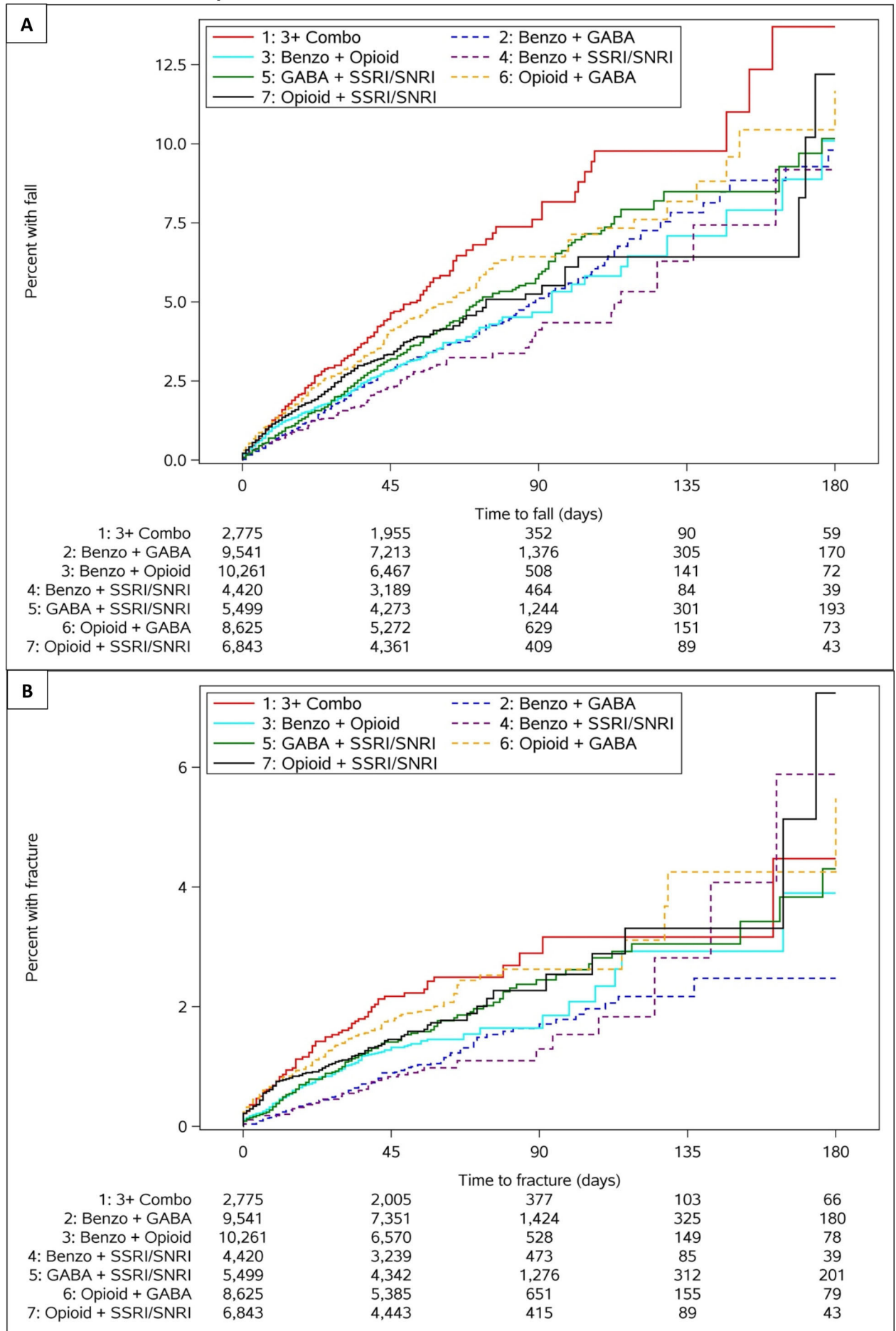

Figure 1 Time to the incidence of (A) fall and (B) fracture after initiation of different drug combinations from KaplanMeier estimator. SSRI/SNRI, selective serotonin reuptake inhibitor/serotonin and norepinephrine reuptake inhibitor. GABA, gabapentinoid. 
presents the curves for fracture (benzo +opioid: 3.9\% at 6 months; benzo +GABA: $2.5 \%$; benzo +SSRI/SNRI: $5.9 \%$; opioid +GABA: $5.5 \%$; opioid +SSRI/SNRI: $7.2 \%$; GABA +SSRI/SNRI: $4.3 \%$; $\geq 3$ drugs: $4.5 \%$ ).

\section{Fall risk}

Table 2 presents the association between drug combination and fall risk as adjusted HRs from the multivariable model adjusted for demographic characteristics, chronic conditions and history of fall and hospitalisation in the prior year. Regimens with a combination of $\geq 3$ drugs (aHR, 1.38; 95\% CI 1.14 to 1.67 ) and opioid +GABA (aHR, 1.18; $95 \%$ CI 1.02 to 1.37 ) were associated with a higher risk of falls, compared with use of benzo +opioid. Characteristics that conferred an increased fall risk included older age (75-84: aHR, 1.36; 95\% CI 1.22 to 1.52; $\geq 85$ : aHR, 1.74; $95 \%$ CI 1.51 to 2.00 ) and Medicare entitlement due to age (aHR, 1.14; 95\% CI 1.02 to 1.27). History of fall (aHR, 2.48; $95 \%$ CI 2.25 to 2.75 ) and hospitalisation (aHR, 1.20; $95 \%$ CI 1.08 to 1.33 ) in the prior year were associated with a higher fall risk. Multiple comorbid conditions were also associated with increased fall risk: Alzheimer's/dementia (aHR, 1.37; 95\% CI 1.24 to 1.52), cancer (aHR, 1.18; 95\% CI 1.06 to 1.30 ), CKD (aHR, 1.20 ; $95 \%$ CI 1.09 to 1.33), COPD (aHR, 1.16; 95\% CI 1.04 to 1.29), diabetes (aHR, 1.18; 95\% CI 1.02 to 1.35 ) and history of drug use disorder (aHR, 1.24; $95 \%$ CI 1.03 to 1.49 ).

\section{Fracture risk}

Table 3 presents the association between drug combination and fracture risk from the multivariable model adjusted for demographic characteristics, chronic conditions and history of fracture and hospitalisation in the prior year. The use of benzo +GABA (aHR, 0.76; 95\% CI 0.59 to 0.98 ) maintained a lower fracture risk compared with benzo +opioiduse. Older age (75-84: aHR, 1.23; 95\% CI 1.02 to 1.46), history of fracture (aHR, 5.18; $95 \%$ CI 4.36 to 6.15 ) and hospitalisation (aHR, 1.40; $95 \% \mathrm{CI} 1.18$ to 1.66 ) in the prior year were associated with an increased risk of fracture. Comorbid conditions associated with an increased fracture risk were Alzheimer's/ dementia (aHR, 1.27; 95\% CI 1.08 to 1.51), cancer (aHR, 1.27; 95\% CI 1.08 to 1.49 ), CKD (aHR, 1.32; 95\% CI 1.13 to 1.55) and history of drug use disorder (aHR, 1.35; $95 \%$ CI 1.03 to 1.77 ).

\section{Interactions}

Among the tested interactions, only the interaction of drug combination and Alzheimer's/dementia was statistically significant $(\mathrm{p}=0.0023)$ for the outcome of fall (online supplemental table 5). After stratifying by Alzheimer's/dementia status, $\geq 3$ drugs (aHR, 1.71; 95\% CI 1.22 to 2.39), benzo +GABA (aHR, 1.53; 95\% CI 1.16 to 2.02) and opioid +GABA (aHR, 1.41; 95\% CI 1.07 to 1.87 ) were associated with a higher risk of fall in patients with this comorbidity compared with the opioid +benzo combination; in individuals without Alzheimer's dementia, no combinations were associated with a higher fall risk compared with the benzo +opioid combination.

\section{IPTW propensity score analysis}

IPTW with propensity score was used to limit the impact of confounding by indication across our study groups. Absolute standardised differences were used to assess balance across treatment groups after weighting and are presented in online supplemental table 6 . The maximum absolute standardised differences across groups are displayed in figure 2, with all values estimated below 0.1 . The results from the propensity score model for fall are presented in table 2, and the results for fracture are presented in table 3 . The results from this model were consistent with the multivariable adjusted model for the combination use of $\geq 3$ drug classes and opioid +GABA for the outcome of fall. Results of benzo +GABA were not significant for predicting a lower risk of fracture, as in the multivariable model.

\section{Sensitivity analyses}

Two sets of sensitivity analyses were conducted to test the robustness of findings from the multivariable model. First, excluding patients with cancer from the multivariable analysis showed the combination of $\geq 3$ drug classes predicting a higher risk of fall and fracture compared with the benzo +opioid combination (online supplemental table 7). Additionally, the combination of benzo +GABA was associated with a lower risk of fracture, consistent with the fully adjusted multivariable model in the main analyses. Second, excluding patients with any drug combination in the prior 3 months to the initiation of a new combination found that the combination of opioid +GABA was associated with a higher risk of fall and fracture compared with the combination of benzo +opioid (online supplemental table 7). Online supplemental table 8 presents the demographic and clinical characteristics of the full study cohorts for our original analyses, of the model excluding patients with cancer, and of the model with the 3-month lookback period.

\section{DISCUSSION}

We examined associations between different combinations of opioid, benzodiazepine, gabapentinoid and SSRI/SNRI classes of medications and the risk of falls and fractures among Medicare beneficiaries with co-existing chronic pain and anxiety disorders. We found that-regardless of medication class-co-prescribing three or more psychotropic medications was associated with increased risk of falls in this population of patients. This finding is consistent with prior research showing an association of psychotropic polypharmacy and greater morbidity in older adults. ${ }^{33}{ }^{34}$ The magnitude of the association between co-prescribing of $\geq 3$ psychotropics and the risk of falls/fractures is significantly higher in those with Alzheimer's disease and other dementias compared with those without, a finding consistent with research 
Table 2 Association of drug combination with falls

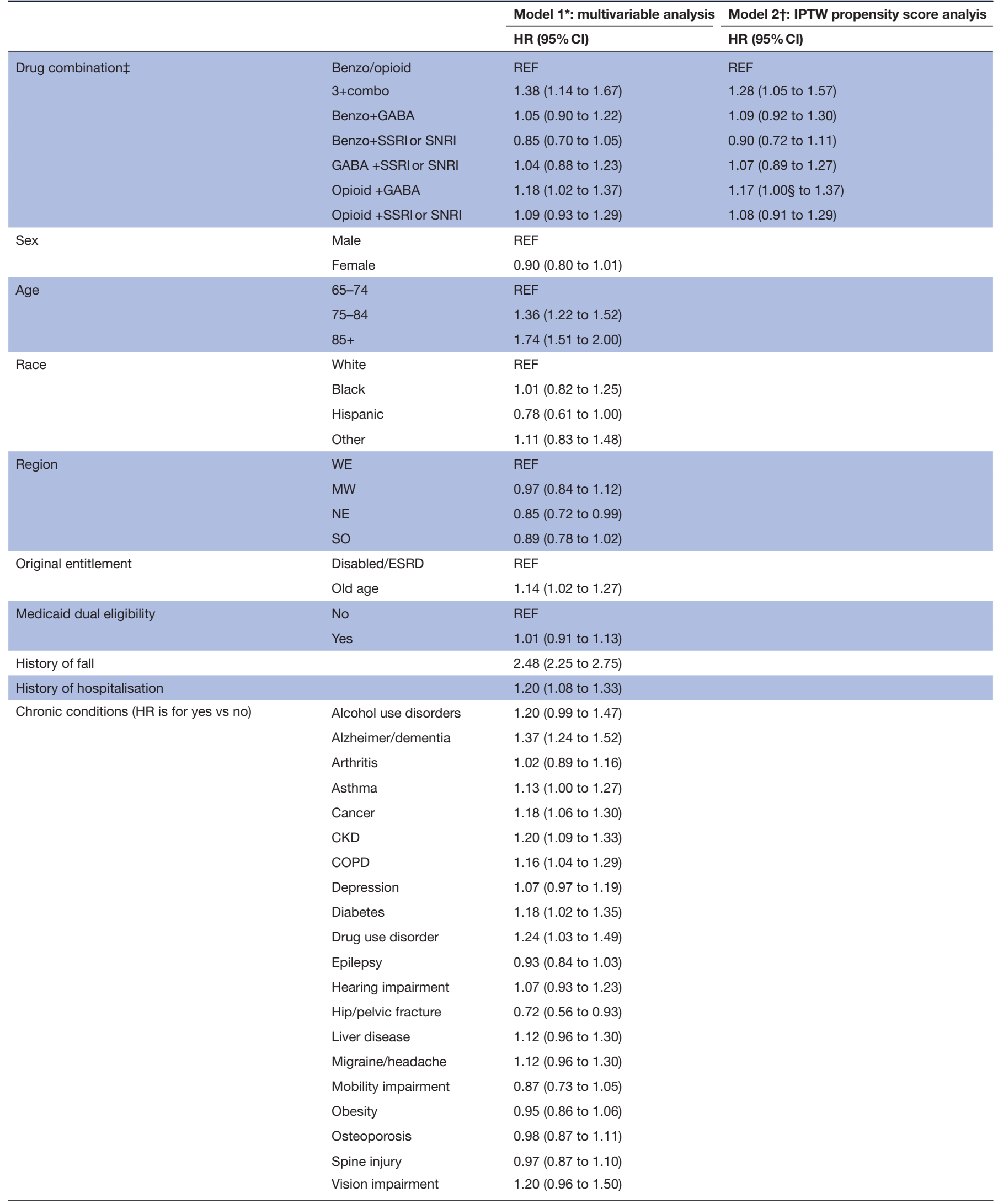


${ }^{*}$ The multivariable analysis was adjusted for demographic variables, history of fall, history of hospitalisation and chronic conditions. †Inverse probability of treatment weighting with propensity score.

$\ddagger$ The $p$ value for the overall drug combination variable is 0.0011 for the fall models.

§The $p$ value for the opioid +GABA combination in the propensity model is 0.0492 and the lower bound is 1.001 .

CKD, chronic kidney disease; COPD, chronic obstructive pulmonary disease; ESRD, End-stage renal disease; GABA, gabapentinoid; IPTW, inverse probability of treatment weighting; MW, Midwest; NE, Northeast; REF, reference group; SNRI, serotonin-norepinephrine reuptake inhibitor; SO, South; SSRI, selective serotonin reuptake inhibitor; WE, West.

showing amplified toxicity of couse of CNS-active medications in persons with dementia disorders. ${ }^{35}$

We also found opioid +GABA co-prescribing was independently associated with a greater risk of falls compared with opioid + benzo co-prescribing, a finding that persisted in the IPTW propensity score analysis. This finding is unexpected, as past research has shown associations of opioid + benzo co-prescribing with high rates of falls, fractures and deaths. ${ }^{459-1336}$ It is unclear why opioid +GABA demonstrated higher fall risk than opioid + benzo, given that, alone or in combinations, all CNS-active agents (opioid, GABA and benzo) are associated with impaired alertness and motor response, which increase fall and fracture risk. ${ }^{37-40}$ One possible explanation is that opioid +GABA users likely have more pain conditions (eg, knee, back or hip pain, peripheral neuropathy) that may affect mobility, balance and strength, thus further elevating the risk of falls, in addition to the fall risks from the CNS depressant effects of opioid +GABA. Yet, the higher fall risk remains significant in our analysis after adjusting for pain conditions, suggesting the possibility of other unmeasured fall-associated factors when opioids and GABA are used in combination. It is possible that the sedating effects of gabapentinoids, particularly in combination with opioids, are being underestimated by clinicians, and that this benzodiazepine-sparing combination is not inherently safer than the combination of opioids and benzodiazepines for the outcome of fall.

Our finding of decreased fracture risk with the benzo +GABA combination from the multivariable model was not robust and was inconsistent with the IPTW propensity score analysis, possibly a reflection of unmeasured variables that may influence likelihood of the complex outcome of fracture. Future research is needed to inform safe and effective combinations of medications in patients regarding fracture risk, possibily in more narrowly selected groups of patients.

For the sensitivity analysis that excludes patients with a combination in the prior 3 months, versus 1 month, as in the main analysis, the results are different compared with the fully adjusted multivariable model: the combination of opioid +GABA predicts a higher risk of both fall and fracture versus the combination of benzo +opioid. In addition to a halving of the sample size, it is possible that, compared with the main analysis' cohort, the demographic and clinical differences in this group of patients may be responsible for the differences in risk of fall and fracture. For example, patients in this group had older age distributions and lower rates of depression, drug use disorder and obesity. The duration of their combination use was also shorter, and this cohort of patients had a lower proportion of the benzo +opioid and $\geq 3$ drug combinations.

The absence of significant reduction in the risk of falls / fractures between GABA+SSRI/SNRI and other opioidsparing or benzodiazepine-sparing combinations versus opioid +benzo co-prescribing was contrary to our expectations. Recent guidelines and federal and state policies urge against the combination of benzodiazepines and opioids. While this combination is now known to confer a high risk for morbidity and overdose mortality, there is not significant research that informs clinician prescribing of alternative combinations to treat comorbid chronic pain and anxiety. Despite the convention that opioidsparing and benzodiazepine-sparing drug combinations might be safer for patients, emerging research highlights the adverse effects of gabapentinoids, particularly in combination with opioid analgesics. However, it is also important to recognise that both opioids and benzodiazepines, as well as gabapentinoids and SSRI/SNRIs, are important and effective medications in the right clinical contexts. Emerging comparative drug toxicity research should not be used to drive consequential prescribing decisions for patients without carefully weighing the benefits and harms within an individualised patient-centred framework, particularly in those with chronic pain and anxiety disorders. Often, the race to de-prescribe without a circumspect, cautious approach can lead to greater harm for patients. ${ }^{41} 42$

There are several limitations to this study, including its retrospective design and the fact that drug prescriptions do not necessarily indicate their use. This study also did not analyse the association of dose within and across combination drug regimens, but only compared combinations by pharmacologic class. Additionally, confounding by indication is also a limitation of this study, common in observational research of drug effects. While this study seeks to adjust for many clinical factors in the multivariable analysis, and test for robustness using IPTW propensity score analysis, it is possible that the indication for certain combinations was linked to fall or fracture outcomes, rather than outcomes being predicted solely by the combination. Indeed, that many of the statistically significant findings from the multivariable model were insignificant in the IPTW propensity score analysis highlight this point and the underlying challenges untangling adverse effects 
Table 3 Association of drug combination with fracture

\begin{tabular}{|c|c|c|c|}
\hline & & \multirow{2}{*}{$\begin{array}{l}\text { Model } 1^{\star} \text { : multivariable analysis } \\
\text { HR }(95 \% \mathrm{Cl})\end{array}$} & \multirow{2}{*}{$\begin{array}{l}\text { Model 2†: IPTW propensity score analysis } \\
\text { HR }(95 \% \mathrm{Cl})\end{array}$} \\
\hline & & & \\
\hline \multirow[t]{7}{*}{ Drug combination $\ddagger$} & Benzo/opioid & REF & REF \\
\hline & $3+$ combo & 1.30 (0.97 to 1.74$)$ & 1.12 (0.82 to 1.53$)$ \\
\hline & Benzo+GABA & 0.76 (0.59 to 0.98$)$ & $0.82(0.62$ to 1.09$)$ \\
\hline & Benzo+SSRI or SNRI & 0.73 (0.52 to 1.02$)$ & 0.75 (0.53 to 1.06$)$ \\
\hline & GABA +SSRI or SNRI & 1.05 (0.81 to 1.36$)$ & 1.09 (0.83 to 1.43$)$ \\
\hline & Opioid +GABA & 1.19 (0.94 to 1.49$)$ & 1.15 (0.90 to 1.45$)$ \\
\hline & Opioid +SSRI or SNRI & $1.12(0.88$ to 1.44$)$ & 1.17 (0.89 to 1.53$)$ \\
\hline \multirow[t]{2}{*}{ Sex } & Male & REF & \\
\hline & Female & 0.85 (0.70 to 1.02$)$ & \\
\hline \multirow[t]{3}{*}{ Age } & $65-74$ & REF & \\
\hline & $75-84$ & 1.23 (1.03 to 1.46$)$ & \\
\hline & $85+$ & 1.22 (0.98 to 1.53$)$ & \\
\hline \multirow[t]{4}{*}{ Race } & White & REF & \\
\hline & Black & 0.97 (0.67 to 1.40$)$ & \\
\hline & Hispanic & 0.76 (0.50 to 1.15$)$ & \\
\hline & Other & $1.29(0.84$ to 1.99$)$ & \\
\hline \multirow[t]{4}{*}{ Region } & WE & REF & \\
\hline & MW & 0.69 (0.54 to 0.87$)$ & \\
\hline & NE & 0.99 (0.78 to 1.24$)$ & \\
\hline & SO & 0.77 (0.63 to 0.94$)$ & \\
\hline \multirow[t]{2}{*}{ Original entitlement } & Disabled/ESRD & REF & \\
\hline & Old age & $1.06(0.89$ to 1.26$)$ & \\
\hline \multirow[t]{2}{*}{ Medicaid dual eligibility } & No & REF & \\
\hline & Yes & $0.85(0.72$ to 1.01$)$ & \\
\hline History of fracture & & 5.18 (4.36 to 6.15$)$ & \\
\hline History of hospitalisation & & 1.40 (1.18 to 1.66$)$ & \\
\hline \multirow{20}{*}{$\begin{array}{l}\text { Chronic conditions (HR is for yes } \\
\text { vs no) }\end{array}$} & Alcohol use disorders & 1.03 (0.76 to 1.41$)$ & \\
\hline & Alzheimer/dementia & 1.27 (1.08 to 1.51$)$ & \\
\hline & Arthritis & 0.83 (0.67 to 1.03$)$ & \\
\hline & Asthma & 1.04 (0.86 to 1.26$)$ & \\
\hline & Cancer & 1.27 (1.08 to 1.49 ) & \\
\hline & CKD & 1.32 (1.13 to 1.55$)$ & \\
\hline & COPD & 1.15 (0.97 to 1.36$)$ & \\
\hline & Depression & $0.90(0.77$ to 1.06$)$ & \\
\hline & Diabetes & $1.01(0.81$ to 1.26$)$ & \\
\hline & Drug use disorder & 1.35 (1.03 to 1.77$)$ & \\
\hline & Epilepsy & $0.92(0.79$ to 1.08$)$ & \\
\hline & Hearing impairment & 1.06 (0.85 to 1.32$)$ & \\
\hline & Hip/pelvic fracture & 0.90 (0.66 to 1.21$)$ & \\
\hline & Liver disease & 1.18 (0.93 to 1.49$)$ & \\
\hline & Migraine/headache & 1.01 (0.79 to 1.30$)$ & \\
\hline & Mobility impairment & 0.97 (0.74 to 1.27$)$ & \\
\hline & Obesity & 0.91 (0.77 to 1.08$)$ & \\
\hline & Osteoporosis & 1.18 (0.99 to 1.40$)$ & \\
\hline & Spine injury & 0.95 (0.79 to 1.15$)$ & \\
\hline & Vision impairment & 1.15 (0.87 to 1.53$)$ & \\
\hline
\end{tabular}

*The multivariable analysis was adjusted for demographic variables, history of fracture, history of hospitalisation and chronic conditions.

tInverse probability of treatment weighting with propensity score.

$\ddagger$ The $p$ value for the overall drug combination variable is 0.0008 for the fracture models.

CKD, chronic kidney disease; COPD, chronic obstructive pulmonary disease; ESRD, end-stage renal disease; GABA, gabapentinoid; IPTW, inverse probability of treatment weighting;

MW, Midwest; NE, Northeast; REF, reference group; SNRI, serotonin-norepinephrine reuptake inhibitor; SO, South; SSRI, selective serotonin reuptake inhibitor; WE, West. 


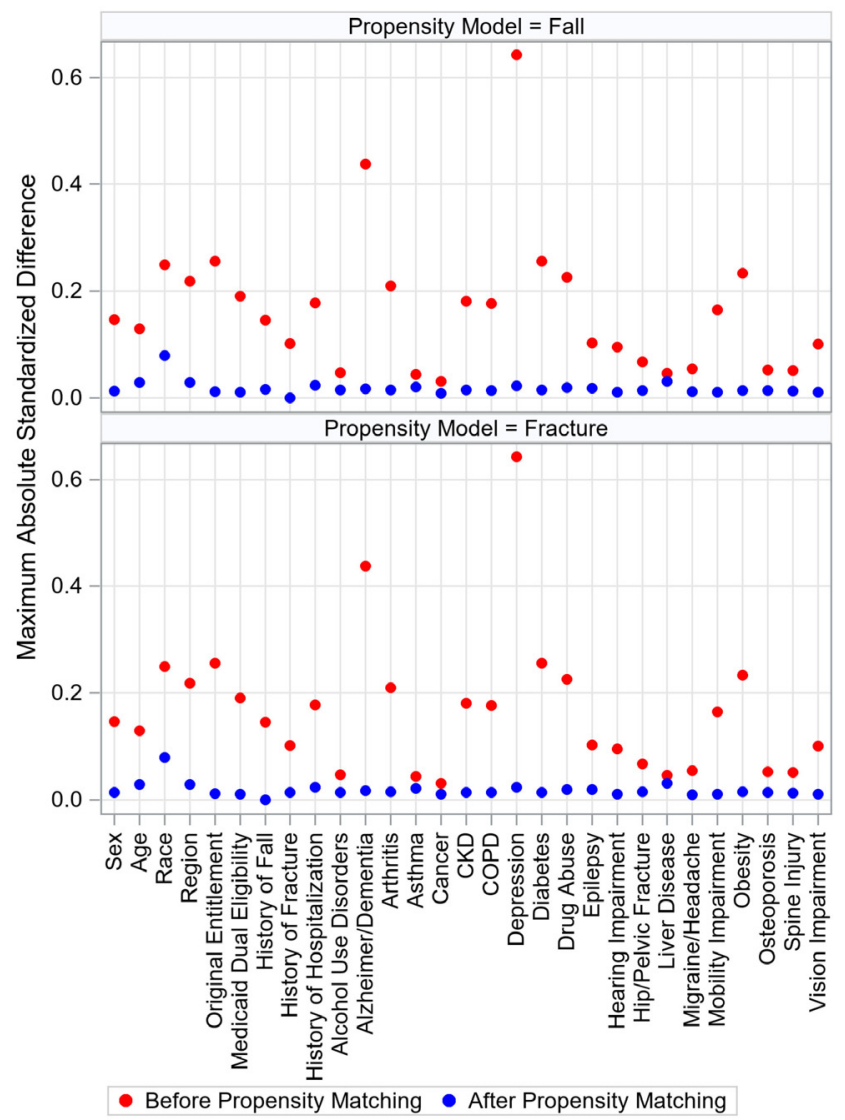

Figure 2 Maximum absolute standardised differences before and after inverse probability of treatment weighing with propensity score for the fall and fracture mode. CKD, chronic kidney disease; COPD, chronic obstructive pulmonary disease. CKD, chronic kidney disease. COPD, chronic obstructive pulmonary disease.

of drugs that may have different use cases, by approved indication or by real-life use pattern. Prescribers may be modifying their psychotropic prescribing practices to reflect their clinical perceptions of patients' risk of side effects such that high-fall risk patients may be given SSRI/SNRIs instead of benzodiazepines for anxiety, for example. In this vein, this study did not adjust severity in chronic pain or anxiety conditions, a factor that may influence clinician prescribing as well. Claims data may not capture such severity-based adjustment of choice and dosing of medications for pain and anxiety conditions, but this is an area for future quantitative and qualitative study. Finally, uncaptured and unmeasured differences in characteristics across patients being prescribed different drug regimens may explain predisposition to fall or fracture, beyond exposure to combination prescriptions.

In sum, this study found that the prescribing of $\geq 3$ classes of medications and opioid + GABA co-prescribing were associated with a higher fall risk than opioid + benzo co-prescribing in patients with co-occurring chronic pain and anxiety. Our findings add to the body of research on the comparative toxicity profiles of different combinations of psychoactive medications (GABAs, SSRIs and
SNRIs) commonly used with or as substitutes for opioids or benzodiazepines in patients with chronic pain and anxiety disorders. Our findings may help clinicians weigh benefits and harms when prescribing drug combinations, especially in older patients with chronic pain and comorbid anxiety disorders, conditions that commonly co-occur in clinical practice. More research is required on the compound effects of multiple CNS-active agents on morbidity and mortality in older patients, particularly in the chronic use setting.

\section{Author affiliations}

${ }^{1}$ Department of Medicine, Vanderbilt University Medical Center, Nashville, Tennessee, USA

${ }^{2}$ Division of Geriatrics and Palliative Medicine, Department of Internal Medicine, The University of Texas Medical Branch at Galveston, Galveston, Texas, USA

${ }^{3}$ Sealy Center on Aging, The University of Texas Medical Branch at Galveston, Galveston, Texas, USA

${ }^{4}$ Office of Biostatistics, Department of Preventive Medicine and Population Health, The University of Texas Medical Branch at Galveston, Galveston, Texas, USA ${ }^{5}$ Department of Preventive Medicine and Population Health, The University of Texas Medical Branch at Galveston, Galveston, Texas, USA

\section{Twitter Rahul Shah @RahulShahK}

Contributors Y-FK and JW had full access to all of the data in this study and take full responsibility for the integrity of the data and accuracy of the data analysis. Concept and design: MAR, Y-FK. Acquisition, analysis or interpretation of the data: RS, MAR, JW, Y-FK. Drafting of the manuscript: RS, MAR, JW, Y-FK. Critical revision of the manuscript for important intellectual content: RS, MAR, JW, Y-FK. Statistical analysis: Y-FK, JW. Obtained funding: MAR, Y-AK. Administrative, technical or material support: Y-FK, JW. Supervision: Y-FK, MAR. Y-FK is responsible for the overall content of this study as the guarantor.

Funding This work was supported by grants R01DA039192 from the National Institute on Drug Abuse and P3OAG024832 from the National Institute on Aging.

Competing interests All authors have completed the ICMJE uniform disclosure form at www.icmje.org/coi_disclosure.pdf and declare: Raji and Kuo were recipients of the grant funding that supported this work from the US National Institute on Drug Abuse and National Institute on Aging; no financial relationships with any organisation that might have an interest in the submitted work in the previous 3 years; no other relationships or activities that could appear to have influenced the submitted work.

Patient consent for publication Not applicable.

Ethics approval The institutional review board of the University of Texas Medical Branch approved this study.

Provenance and peer review Not commissioned; externally peer reviewed.

Data availability statement Data may be obtained from a third party and are not publicly available. Data may be obtained from a third party and are not publicly available. The data used in this study are deidentified Medicare claims data, which may be provided to researchers upon request from the Centers for Medicare and Medicaid Services of the US.

Supplemental material This content has been supplied by the author(s). It has not been vetted by BMJ Publishing Group Limited (BMJ) and may not have been peer-reviewed. Any opinions or recommendations discussed are solely those of the author(s) and are not endorsed by BMJ. BMJ disclaims all liability and responsibility arising from any reliance placed on the content. Where the content includes any translated material, BMJ does not warrant the accuracy and reliability of the translations (including but not limited to local regulations, clinical guidelines, terminology, drug names and drug dosages), and is not responsible for any error and/or omissions arising from translation and adaptation or otherwise.

Open access This is an open access article distributed in accordance with the Creative Commons Attribution Non Commercial (CC BY-NC 4.0) license, which permits others to distribute, remix, adapt, build upon this work non-commercially, and license their derivative works on different terms, provided the original work is properly cited, appropriate credit is given, any changes made indicated, and the use is non-commercial. See: http://creativecommons.org/licenses/by-nc/4.0/. 
ORCID iDs

Rahul Shah http://orcid.org/0000-0001-6187-4690

Mukaila A Raji http://orcid.org/0000-0002-7460-7281

\section{REFERENCES}

1 Wilson N. Drug and Opioid-Involved overdose deaths - United States, 2017-2018. MMWR Morb Mortal Wkly Rep 2020;69.

2 Understanding the Epidemic. Drug overdose / CDC injury center 2020.

3 CDC. Annual surveillance report of drug-related risks and outcomes, 2019.

4 Gladden RM. Changes in opioid-involved overdose deaths by opioid type and presence of benzodiazepines, cocaine, and methamphetamine - 25 states, July-December 2017 to JanuaryJune 2018. MMWR Morb Mortal Wkly Rep 2019:68.

5 Jones CM, McAninch JK. Emergency department visits and overdose deaths from combined use of opioids and benzodiazepines. Am J Prev Med 2015;49:493-501.

6 Gomez AF, Barthel AL, Hofmann SG. Comparing the efficacy of benzodiazepines and serotonergic anti-depressants for adults with generalized anxiety disorder: a meta-analytic review. Expert Opin Pharmacother 2018;19:883-94.

7 Hirschtritt ME, Delucchi KL, Olfson M. Outpatient, combined use of opioid and benzodiazepine medications in the United States, 19932014. Prev Med Rep 2018;9:49-54.

8 Rhee TG. Coprescribing of benzodiazepines and opioids in older adults: rates, correlates, and national trends. J Gerontol A Biol Sci Med Sci 2019;74:1910-5.

9 Sun EC, Dixit A, Humphreys K, et al. Association between concurrent use of prescription opioids and benzodiazepines and overdose: retrospective analysis. BMJ 2017;356:j760.

10 Asmundson GJG, Katz J. Understanding the co-occurrence of anxiety disorders and chronic pain: state-of-the-art. Depress Anxiety 2009;26:888-901.

11 Boon M, van Dorp E, Broens S, et al. Combining opioids and benzodiazepines: effects on mortality and severe adverse respiratory events. Ann Palliat Med 2020;9:542-57.

12 Jann M, Kennedy WK, Lopez G. Benzodiazepines: a major component in unintentional prescription drug overdoses with opioid analgesics. J Pharm Pract 2014;27:5-16.

13 Nurminen J, Puustinen J, Piirtola M, et al. Opioids, antiepileptic and anticholinergic drugs and the risk of fractures in patients 65 years of age and older: a prospective population-based study. Age Ageing 2013;42:318-24.

14 Musich S, Wang SS, Slindee LB, et al. Concurrent use of opioids with other central nervous System-Active medications among older adults. Popul Health Manag 2020;23:286-96.

15 CDC guideline for prescribing opioids for chronic pain - United States, 2016. MMWR Recomm Rep 2016;65.

16 U.S. Food and Drug Administration. FDA warns about serious risks and death when combining opioid pain or cough medicines with benzodiazepines; requires its strongest warning. Drug safety communications, 2017. Available: https://www.fda.gov/drugs/drugsafety-and-availability/fda-drug-safety-communication-fda-warnsabout-serious-risks-and-death-when-combining-opioid-pain-or [Accessed 5 Oct 2020].

17 Jeffery MM, Hooten WM, Jena AB, et al. Rates of physician coprescribing of opioids and benzodiazepines after the release of the centers for disease control and prevention guidelines in 2016. JAMA Netw Open 2019;2:e198325.

18 Bohnert ASB, Guy GP, Losby JL. Opioid prescribing in the United States before and after the centers for disease control and prevention's 2016 opioid guideline. Ann Intern Med 2018;169:367-75.

19 Goodman CW, Brett AS. Gabapentin and pregabalin for pain - is increased prescribing a cause for concern? N Engl J Med Overseas Ed 2017;377:411-4.
20 Goodman CW, Brett AS. Gabapentinoids for pain: potential unintended consequences. Am Fam Physician 2019;100:672-5.

21 Johansen ME. Gabapentinoid use in the United States 2002 through 2015. JAMA Intern Med 2018;178:292-4.

22 Peckham AM, Evoy KE, Ochs L, et al. Gabapentin for offlabel use: evidence-based or cause for concern? Subst Abuse 2018:12:1178221818801311

23 Feltner D, Wittchen $\mathrm{H}-\mathrm{U}$, Kavoussi R, et al. Long-term efficacy of pregabalin in generalized anxiety disorder. Int Clin Psychopharmacol 2008;23:18-28.

24 Lavigne JE, Heckler C, Mathews JL, et al. A randomized, controlled, double-blinded clinical trial of gabapentin 300 versus $900 \mathrm{Mg}$ versus placebo for anxiety symptoms in breast cancer survivors. Breast Cancer Res Treat 2012;136:479-86.

25 Randolph AC, Lin Y-L, Volpi E, et al. Tricyclic antidepressant and/or $\gamma$-aminobutyric Acid-Analog use is associated with fall risk in diabetic peripheral neuropathy. J Am Geriatr Soc 2019;67:1174-81.

26 Gomes T, Greaves S, van den Brink W, et al. Pregabalin and the risk for opioid-related death: a nested case-control study. Ann Intern Med 2018;169:732-4.

27 Longo LP, Johnson B. Addiction: Part I. Benzodiazepines--side effects, abuse risk and alternatives. Am Fam Physician 2000;61:2121.

28 Gomez AF, Barthel AL, Hofmann SG. Comparing the efficacy of benzodiazepines and serotonergic anti-depressants for adults with generalized anxiety disorder: a meta-analytic review. Expert Opin Pharmacother 2018;19:883-94.

29 Slee A, Nazareth I, Bondaronek P. Pharmacological treatments for generalised anxiety disorder: a systematic review and network meta-analysis [published correction appears in Lancet. 2019 Apr 27;393(10182):1698]. Lancet 2019;393:768-77.

30 Riediger C, Schuster T, Barlinn K, et al. Adverse effects of antidepressants for chronic pain: a systematic review and metaanalysis. Front Neurol 2017;8:307.

31 Kremer M, Salvat E, Muller A, et al. Antidepressants and gabapentinoids in neuropathic pain: mechanistic insights. Neuroscience 2016;338:183-206.

32 Centers for medicare \& medicaid services chronic condition data warehouse: chronic condition categories. Available: https://www2. ccwdata.org/web/guest/condition-categories [Accessed 1 Mar 2020].

33 Maust DT, Gerlach LB, Gibson A, et al. Trends in central nervous system-active polypharmacy among older adults seen in outpatient care in the United States. JAMA Intern Med 2017;177:583-5.

34 Shmuel S, Lund JL, Alvarez C, et al. Polypharmacy and incident frailty in a longitudinal community-based cohort study. J Am Geriatr Soc 2019;67:2482-9.

35 Borda MG, Jaramillo-Jimenez A, Oesterhus R. Benzodiazepines and antidepressants: effects on cognitive and functional decline in Alzheimer's disease and Lewy body dementia [published online ahead of print, 2020 Dec 31]. Int J Geriatr Psychiatry. 2020.

36 Park TW, Saitz R, Ganoczy D, et al. Benzodiazepine prescribing patterns and deaths from drug overdose among US veterans receiving opioid analgesics: case-cohort study. BMJ 2015;350:h2698.

37 Saunders KW, Dunn KM, Merrill JO, et al. Relationship of opioid use and dosage levels to fractures in older chronic pain patients. $J$ Gen Intern Med 2010;25:310-5.

38 Kinjo M, Setoguchi S, Schneeweiss S, et al. Bone mineral density in subjects using central nervous system-active medications. Am J Med 2005;118:1414.e7-e12.

39 Yue Q, Ma Y, Teng Y, et al. An updated analysis of opioids increasing the risk of fractures. PLoS One 2020;15:e0220216.

40 Takkouche B, Montes-Martínez A, Gill SS, et al. Psychotropic medications and the risk of fracture: a meta-analysis. Drug Saf 2007;30:171-84.

41 Dowell D, Haegerich T, Chou R. No shortcuts to safer opioid prescribing. N Engl J Med 2019;380:2285-7.

42 Hirschtritt ME, Olfson M, Kroenke K. Balancing the risks and benefits of benzodiazepines. JAMA 2021;325:347-8. 